\title{
The Effects of BADGE and Caffeine on the Time-Course Response of Adiponectin and Lipid Oxidative Enzymes in High Fat Diet-Fed C57BL/6J Mice: Correlation with Reduced Adiposity and Steatosis
}

\author{
Jun-Won YUN ${ }^{1)}$, Eui-Seok SHIN ${ }^{1)}$, Si-Young $\mathrm{CHO}^{1)}$, Shin-Hyoung $\mathrm{KIM}^{1)}$, \\ Chae-Wook KIM ${ }^{1)}$, Tae-Ryong LEE $^{1)}$, and Bae-Hwan KIM ${ }^{2}$ \\ ${ }^{1)}$ Amorepacific Corporation R\&D Center, 314-1 Bora-dong, Giheung-gu, Yongin-si, Gyeonggi-do \\ 446-729 and ${ }^{2)}$ Department of Public Health, Keimyung University, Daegu 704-701, Korea
}

\begin{abstract}
Adiponectin, which is expressed exclusively in adipose tissue, has been shown to increase fatty acid oxidation via activation of AMP-activated kinase (AMPK) and phosphorylation of acetyl CoA carboxylase (ACC). ACC phosphorylation and carnitine palmitoyl-transferase-1 (CPT1) activity have been shown to be rate controlling factors in fatty acid oxidation. In high fat diet (HFD)-induced obese mice, we analyzed the time-course of changes in the expression of adiponectin and lipid oxidative enzymes induced by treatment with bisphenol A diglycidyl ether (BADGE) or caffeine for 8 weeks, and investigated whether the changes of adiponectin and lipid oxidative enzymes expression correlated with reduced adiposity or steatosis after 8 weeks of treatment. After 8 weeks of treatment, BADGE and caffeine had reduced body weight and epididymal adipose tissue weight in mice fed HFD, and markedly reduced the number of fatty droplets in the liver. Interestingly, the expression of adiponectin and lipid oxidative enzymes significantly increased after 2 weeks of treatment. These results indicate that the expression of adiponectin and lipid oxidative enzymes in the early stages of BADGE or caffeine treatment correlated well with the long-term anti-obesity effects.
\end{abstract}

Key words: adiponectin, bisphenol A diglycidyl ether (BADGE), caffeine, carnitine palmitoyltransferase-1 (CPT1), phospho-acetyl CoA carboxylase (pACC)

\section{Introduction}

Bisphenol A diglycidyl ether (BADGE) is a peroxisome proliferator-activated receptor gamma (PPAR gamma) antagonist and has been shown to have antiobesity and anti-diabetic effects in mice fed high fat diet (HFD) [24]. Caffeine reduces the body fat mass of
Sprague-Dawley rats fed a HFD and noninsulin-dependent diabetic KK mice $[9,11]$. The model of HFD-induced obesity is also useful for studying obesity, and is often used for searching for potential anti-obesity chemicals. Chronic exposure to HFD markedly increases adipose tissue weight compared to a low fat diet (LFD) [6]. Surwit et al. first described an animal model of

(Received 19 May 2008 / Accepted 7 July 2008)

Address corresponding: B.-H. Kim, Department of Public Health, Keimyung University, Daegu 704-701, Korea

J.-W. Yun and E.-S. Shin equally contributed to this work. 
Table 1. Formulation of the AIN93G diet

\begin{tabular}{lcc}
\hline \multicolumn{1}{c}{ Ingredient } & $\begin{array}{c}\text { AIN93G } \\
(\mathrm{g} / \mathrm{kg} \text { of diet })\end{array}$ & $\begin{array}{c}\text { AIN93G (40\% HFD) } \\
(\mathrm{g} / \mathrm{kg} \text { of diet })\end{array}$ \\
\hline Cornstarch & 397.486 & 48.186 \\
Casein & 200 & 200 \\
Dextrose & 132 & 132 \\
Sucrose & 100 & 100 \\
Soybean oil & 70 & 70 \\
Shortening & 0 & 330 \\
Cellulose & 50 & 50 \\
Vitamin mix (AIN-93-VX) & 10 & 14.3 \\
Mineral mix (AIN-93G) & 35 & 50 \\
L-cystine & 3 & 3 \\
Choline bitartrate & 2.5 & 2.5 \\
Tert-Butylhydroquinone (TBHQ) & 0.014 & 0.014 \\
\hline
\end{tabular}

long-term HFD-induced obesity in 1988 [18]. This model has since been shown to be most useful in C57BL/6J mice compared to other mouse strains [1, 17, 22].

During oxidation, phosphorylation of acetyl CoA carboxylase (ACC) has been shown to reduce the production of malonyl-CoA, an allosteric inhibitor of carnitine palmitoyl-transferase-1 (CPT1) [13]. A decrease in malonyl-CoA subsequently promotes CPT1 activity and fatty acid oxidation in most tissues [16, 23]. The transfer of long-chain fatty acids across the mitochondrial membrane is mediated by CPT1, which facilitates the coupling of carnitine to fatty acyl-CoA $[14,21]$. The product of this reaction, acylcarnitine, can be transferred across the outer membrane by CPT1. CPT1 activity has been shown to be a rate controlling factor in hepatic oxidation [14]. Adiponectin, which is expressed exclusively in adipose tissue, has been shown to increase fatty acid oxidation via activation of AMP-activated kinase (AMPK) and phosphorylation of ACC [19]. Recently, numerous studies of the relationship of adiponectin and lipid oxidative enzymes with obesity have been taken place. In the HFD-induced obesity model, the identification of adiponectin or lipid oxidative enzymes can also be very beneficial for the analysis of anti-obesity effects.

Observations of the anti-obesity effect of BADGE and caffeine were previously published $[9,11,24]$. However, the effects of BADGE and caffeine on the timecourse responses of adiponectin and lipid oxidative enzymes, such as pACC and CPT1, in the HFD-induced obesity model are still unclear. Therefore, we analyzed the time-course of changes in the expression of adiponectin and lipid oxidative enzymes induced by BADGE or caffeine in HFD-induced obese mice, and compared these results with final body weight and adipose tissue weight changes. Using this data, we investigated whether BADGE- or caffeine-induced changes in adiponectin and lipid oxidative enzymes expression correlated with reduced adiposity or steatosis in HFD-induced obese mice.

\section{Materials and Methods}

\section{Animals and diets}

Male C57BL/6J mice (Samtako Co., Osan, Korea) were given free access to an AIN93G 40\% HFD (Hanlive R\&D Co., Yangju, Korea) or AIN93G diet (Table 1) [15]. Mice were housed in individual cages in a controlled environment $\left(23 \pm 3^{\circ} \mathrm{C}\right.$, and relative humidity of $40-$ $60 \%$ ). Lighting was adjusted automatically to provide a $12 \mathrm{~h}$ light/12h dark cycle. Throughout the study, animals had access ad libitum to tap water. The local water supply authority regularly conducted a water analysis for bacteriologic and chemical contaminants. No contaminants were present in the diets or water at levels that would have interfered with the study. Animal care and the study protocol were carried out in accordance with the guidelines of the Institutional Animal Care and Use Committee (IACUC) of Amorepacific R\&D Center. 
Body weights of C57BL/6J mouse were recorded during the experimental period. Mean daily food intake was calculated every week.

\section{Chemicals}

BADGE and caffeine were obtained from Sigma Aldrich Co. (St Louis, MO, USA). Anti-pACC (phosphorylated on serine 79) antibody was purchased from Cell Signaling Technology (Danvers, MA, USA). Anti-rabbit IgG linked to horseradish peroxidase was supplied by Amersham Bioscience (Piscataway, NJ, USA).

\section{Experimental protocol}

The experiment was performed using 7-week-old male C57BL/6J mice. Mice were divided into the following two groups: the normal control group (NC group) fed an ANI93G diet for 4 weeks ( $n=24)$, and the HFD-induced obesity group (HFD group) fed an AIN93G diet containing $40 \%$ HFD for 4 weeks $(n=72)$. The NC group $(n=24)$ was fed AIN93G for an additional 8 weeks, and the HFD group was randomly assigned to one of the following three diet groups for an additional 8 weeks $(n=24$ for each group): AIN93G 40\% HFD (HFD group), AIN93G $40 \%$ HFD supplemented with $0.03 \%$ BADGE (BADGE group), and AIN93G 40\% HFD supplemented with $0.025 \%$ caffeine (caffeine group). One, two, four, and eight weeks after the start of the supplemented diet, six mice were randomly selected from each group and anesthetized with pentobarbital (50 mg/kg body). Blood was collected via the postvenacava from the anesthetized mice. After blood collection, liver and epididymal white adipose tissue were collected, and the liver was perfused with ice-cold saline. Epididymal white adipose tissue was weighed. Liver and epididymal adipose tissue samples for immunoblot analysis, CPT1 activity assay, and ELISA were frozen immediately in liquid nitrogen. For histological analysis, liver tissue was fixed in $10 \%$ neutral buffered formalin, embedded in paraffin and sectioned into 3-4- $\mu \mathrm{m}$ sections, which were then stained with hematoxylin \& eosin (HE) and examined by light microscopy.

\section{Mouse adiponectin immunoassay}

For a quantitative determination of adiponectin concentrations in epididymal white adipose tissue homoge- nates, a Quantikine immunoassay kit was used (R\&D systems, Minneapolis, USA). The collected epididymal white adipose tissue was rinsed with PBS, homogenized in $5 \mathrm{ml} \mathrm{PBS}$, and stored at $-20^{\circ} \mathrm{C}$ overnight. Homogenates were centrifuged for $5 \mathrm{~min}$ at $5,000 \times \mathrm{g}$ and the supernatants were diluted 2,000-fold. The adiponectin levels were determined according to the method recommended by the manufacturer. A standard curve was obtained in the range of adiponectin from $0.16-10 \mathrm{ng} /$ $\mathrm{ml}$. The adiponectin concentration was calculated from the standard curve and was normalized by determining each total protein concentration.

\section{Immunoblot analysis}

Liver tissues were rinsed in PBS and homogenized on ice in radio-immunoprecipitation assay (RIPA) buffer (pH 8.5) (Sigma Co., St. Louis, USA) with a glass homogenizer. The homogenate was centrifuged to remove cell debris. Fifty micrograms of total protein were separated by $8 \%$ sodium dodecyl sulfate/polyacrylamide gel electrophoresis under reducing conditions; then, the proteins were transferred onto a polyvinylidene difluoride (PVDF) membrane. The membrane was incubated in $5 \%$ non-fat milk in TBS-T $(150 \mathrm{mM} \mathrm{NaCl}, 50 \mathrm{mM}$ Tris-HCl, $0.1 \%$ Tween-20, pH 7.4) for $3 \mathrm{~h}$ to block nonspecific binding. Antibody for pACC was diluted in blocking buffer and then incubated with the membrane overnight in a cold room with shaking. The membranes were washed with TBS-T and incubated with secondary antibodies conjugated to horseradish peroxidase. Immunoreactive proteins were detected by chemiluminescence with an ECL kit (Amersham BioSciences, Piscataway, USA), according to the manufacturer's instructions.

\section{Determination of CPT1 activity in the liver}

Briefly, frozen liver tissue was homogenized and suspended in buffer ( $250 \mathrm{mmol} / 1$ sucrose, $1 \mathrm{mmol} / \mathrm{l}$ EDTA, $10 \mathrm{mmol} / \mathrm{l} \mathrm{HEPES}$ (pH 7.2)). Protease inhibitor cocktail (Sigma Co., St. Louis, USA) was added to the suspension, and it was centrifuged at $600 \times g$ at $4^{\circ} \mathrm{C}$ for $10 \mathrm{~min}$. CPT1 activity in liver tissue was assayed spectrophotometrically according to the method of Karlic et al. [7]. 


\section{Blood analysis}

At the end of the experimental term, blood was collected via the postcaval vein from anesthetized mice. The first appearing blood drop was collected by a sterile cotton pad, and the second blood drop was applied to a glucometer test strip. Blood glucose levels were measured by Glucotrend $2^{\circledR}$ (Boehringer Mannheim/Roche, UK). Next, blood was immediately collected in a vacutainer serum-separating tube (BD Diagnostic, MD, USA). Serum was obtained by centrifugation at 3,000 rpm for $10 \mathrm{~min}$, then stored at $-70^{\circ} \mathrm{C}$ until use. Total cholesterol, high density lipoprotein (HDL)-cholesterol, low density lipoprotein (LDL)-cholesterol, free fatty acid (FFA) and triglyceride (TG) levels were analyzed with an automatic blood chemistry analyzer (Selectra E, Vital Scientific, Netherlands).

\section{Statistical analysis}

All data are represented as the mean \pm standard deviation (SD). Statistical analysis was carried out using Minitab ${ }^{\circledR} 14$ software. The data were subjected to oneway ANOVA followed by post hoc Tukey's multiple range test to determine the effect of treatment and to compare differences among the means of the groups. A value of $P<0.05$ was considered statistically significant; $P<0.1$ was regarded as a tendency.

\section{Results}

Body weight, epididymal fat pad weight, and food intake

The changes in body weight for the four groups during the experimental period are shown in Fig. 1. At 1, 2, and 4 weeks, no differences in the final average body weights of the four groups were detected. At 8 weeks, mice fed HFD had significantly increased body weight compared to the $\mathrm{NC}$ group; however, dietary supplementation with BADGE or caffeine significantly reduced the body weight gain associated with HFD at 8 weeks of supplementation. As a result of this, body weights in the BADGE and the caffeine groups were not significantly different from that of the $\mathrm{NC}$ group after 8 weeks of treatment.

At 8 weeks, the absolute epididymal fat weights of the BADGE- and the caffeine-treated groups were significantly lower than that of the HFD group. Moreover, the

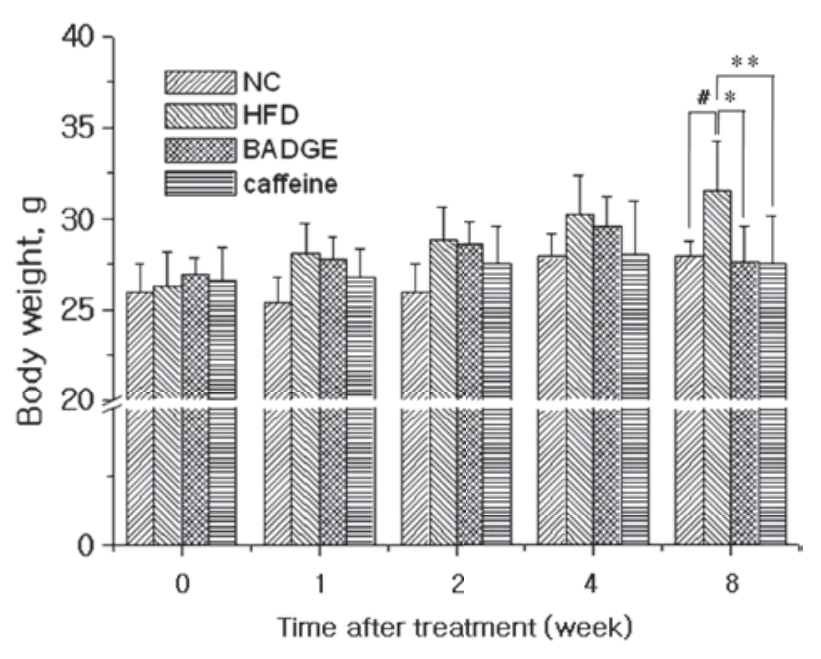

Fig. 1. Body weights of C57BL/6J mice over the 8-week experimental period. NC, normal control; HFD, high-fat diet group; BADGE, HFD+BADGE group; Caffeine, $\mathrm{HFD}+$ caffeine group. Values represent the means $\pm \mathrm{SD}$. The means were compared by one-way ANOVA. ${ }^{\#}, P<0.05$ vs NC group. *, $P<0.05$ vs HFD group. BADGE, bisphenol A diglycidyl ether.

Tabel 2. The epididymal fat weight after 8 weeks of treatment in C57BL/6J mice

\begin{tabular}{lcc}
\hline & \multicolumn{2}{c}{ The epididymal fat weight } \\
\cline { 2 - 3 } & Absolute weight $(\mathrm{g})$ & Relative weight $(\mathrm{g} / 100 \mathrm{~g} \mathrm{BW})$ \\
\hline NC & $0.2405 \pm 0.0348$ & $0.860 \pm 0.101$ \\
HFD & $0.5880 \pm 0.1414^{\#}$ & $1.870 \pm 0.402^{\#}$ \\
BADGE & $0.3302 \pm 0.2178^{*}$ & $1.213 \pm 0.863$ \\
Caffeine & $0.3616 \pm 0.1277^{*}$ & $1.303 \pm 0.491^{* *}$ \\
\hline
\end{tabular}

$\mathrm{NC}$, normal control; HFD, high-fat diet group; BADGE, HFD+BADGE group; Caffeine, HFD+caffeine group. Values represent the means $\pm \mathrm{SD}$. The means were compared by oneway ANOVA. " $P<0.05$ vs NC group. $*, P<0.05$ vs HFD group. $* *, P<0.1$ vs HFD group. BW, body weight; BADGE, bisphenol A diglycidyl ether.

relative epididymal fat weights in both the BADGE and caffeine groups showed a decreasing tendency compared with the HFD group (Table 2). There were no significant differences in the food intake values for the different diet groups over the course of the experiment (Data not shown).

\section{Histological analysis}

Figure 2 shows representative micrographs of liver tissue samples stained with HE. The liver tissue of the 

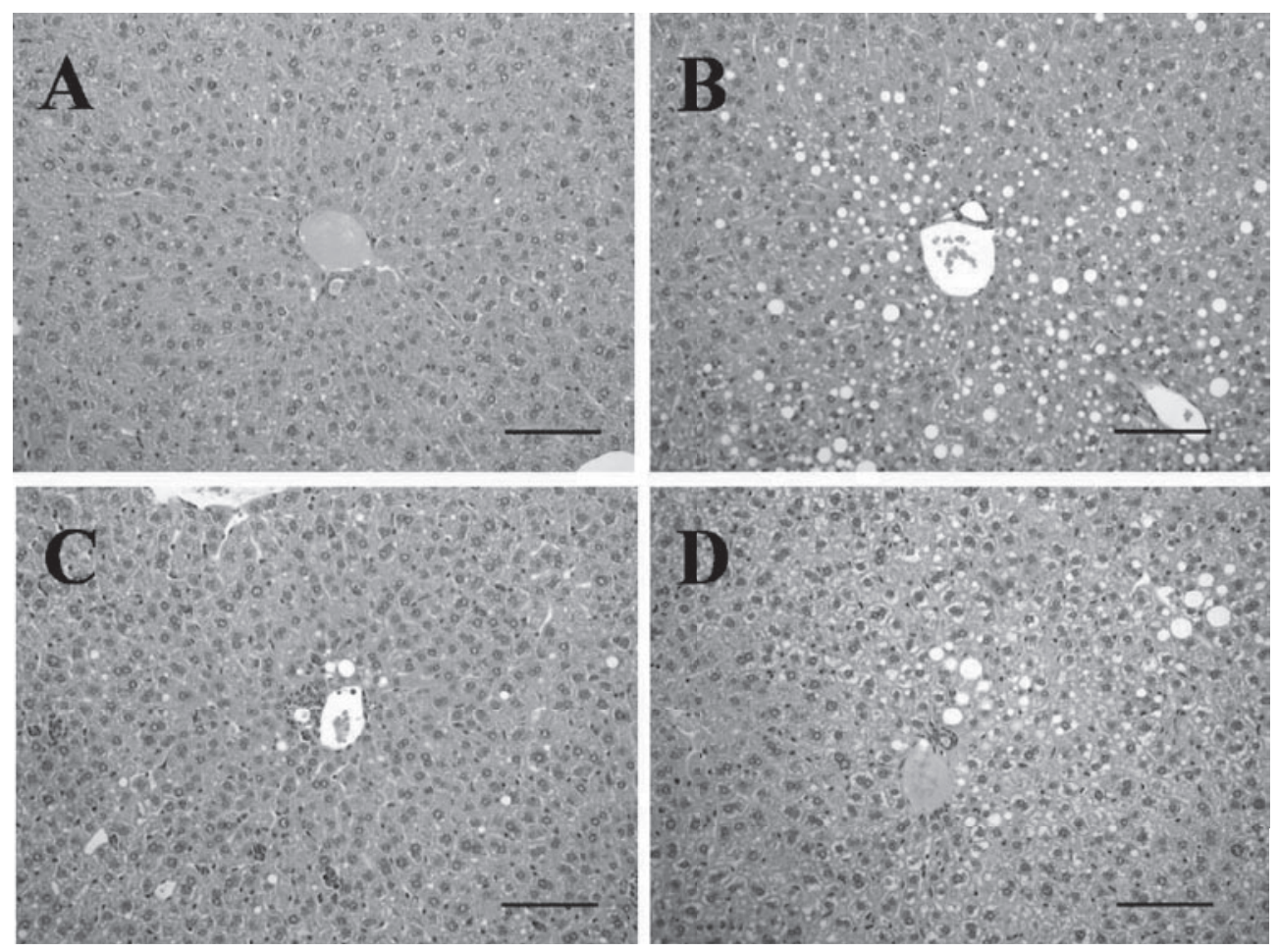

Fig. 2. Representative HE-stained liver tissue after 8 weeks of treament. Liver sections from the NC group (A), HFD group (B), BADGE group (C), and Caffeine group (D) of C57BL/6J mice after 8 weeks of treatment. Liver tissues were stained with hematoxylin-eosin. Bar=100 $\mu \mathrm{m}$.

NC group, which was fed an AIN93G diet for 12 weeks, appeared normal (Fig. 2A). The liver tissue of the HFD group exhibited large macrovacuolar steatosis that extended from the portal space to the centrolobular vein (Fig. 2B), whereas the livers of the BADGE and the caffeine groups showed markedly attenuated hepatic steatosis compared to the HFD group (Figs. 2C and 2D, respectively).

\section{Glucose and serum parameters}

The HFD group showed a significant increase in blood glucose concentration compared to the NC group. Dietary supplementation with BADGE significantly reduced blood glucose levels compared to the HFD group. The caffeine group showed a tendency of decrease in gluose levels compared to the HFD group (Fig. 3). In the BADGE and the caffeine groups, there were no significant differences in total cholesterol, HDL-cholesterol, LDL-cholesterol, and FFA compared to the HFD group (Data not shown).

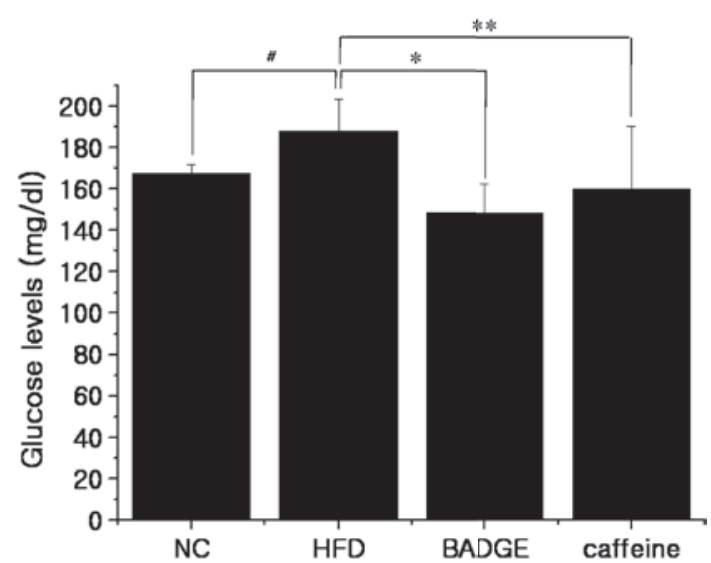

Fig. 3. Glucose levels after 8 weeks of treament. NC, normal control; HFD, high-fat diet group; BADGE, HFD+BADGE group; Caffeine, HFD+caffeine group. Values represent the means $\pm \mathrm{SD}$. The means were compared by one-way ANOVA. ${ }^{\#}, P<0.05$ vs NC group. * $P<0.05$ vs HFD group. $* *, P<0.1$ vs HFD group. BADGE, bisphenol A diglycidyl ether. 


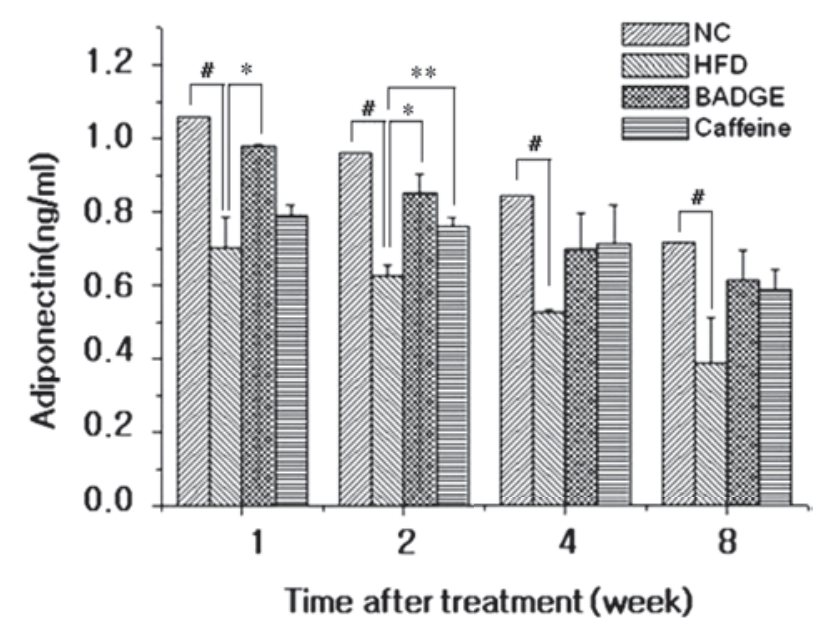

Fig. 4. Expression level of adiponectin in C57BL/6J mice determined by ELISA over the 8-week experimental period. NC, normal control; HFD, high-fat diet group; BADGE, HFD+BADGE group; Caffeine, HFD+caffeine group. Values represent the means $\pm \mathrm{SD}$. The means were compared by one-way ANOVA. ${ }^{\#}, P<0.05$ vs NC group. *, $P<0.05$ vs HFD group. **, $P<0.1$ vs HFD group. $\mathrm{N}=2$, the number of independent experiments. BADGE, bisphenol A diglycidyl ether.

Analysis of adiponectin and lipid oxidative enzymes in HFD-fed mice

We analyzed the changes of the adiponectin and lipid oxidative enzymes, including pACC and CPT1, during the experimental period and compared the levels between groups at the same stage.

Mice fed HFD had significantly decreased adiponectin levels compared to the levels observed in the NC group over the 8-week experimental period. Meanwhile, at 1 and 2 weeks of the supplemented diet regime, the adiponectin level was significantly higher in the BADGE group than in the HFD group $(P<0.05)$. In the caffeine group, the adiponectin level was higher than that of the HFD group at 2 weeks of treatment $(P<0.1)$. At 4 and 8 weeks of treatment, the expression levels of adiponectin in the BADGE and the caffeine groups were slightly higher than in the HFD group, but the significance of this remains unclear (Fig. 4).

We next examined the expression of pACC in the two groups of C57BL/6J mice fed HFD supplemented with BADGE or caffeine using immunoblot analysis. Mice fed HFD showed significantly decreased expression of pACC in comparison to the $\mathrm{NC}$ group at 1,2 , and 4

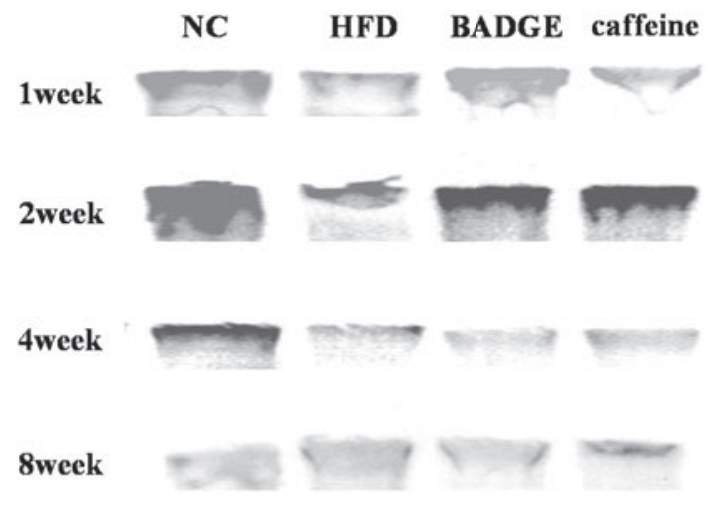

Fig. 5. Expression of pACC in C57BL/6J mice determined by immunoblot analysis over the 8 -week experimental period. NC, normal control; HFD, high-fat diet group; BADGE, HFD+BADGE group; Caffeine, HFD+caffeine group. Representative photos of western blot analyses performed using liver protein samples from NC, HFD, BADGE, and caffeine mice. BADGE, bisphenol A diglycidyl ether.

weeks of supplementation. The expression of pACC in the BADGE group was higher than in the HFD group at 1 and 2 weeks of supplementation. While the pACC level of the caffeine group was similar to that of the HFD group at 1 week, it became higher than that of the HFD group at 2 weeks of supplementation. At 4 and 8 weeks, the expression of pACC in the BADGE and the caffeine groups tended to be similar to the HFD group (Fig. 5).

At 2 weeks of supplementation, the activity of CPT1 in the livers of the HFD group was significantly lower than that of the NC group. In contrast, the activities of CPT1 in the BADGE and the caffeine groups were higher than that of the HFD group (Fig. 6). There were no differences among the groups in the activity of CPT1 at 1, 4, and 8 weeks after beginning treatment (data not shown).

\section{Discussion}

The mice fed HFD supplemented with BADGE or caffeine gained body weight more slowly and had reduced tissue fat weight. Moreover, the BADGE and the caffeine groups showed markedly attenuated hepatic steatosis. We postulate that these effects of BADGE and caffeine might involve enhanced lipid oxidation. 


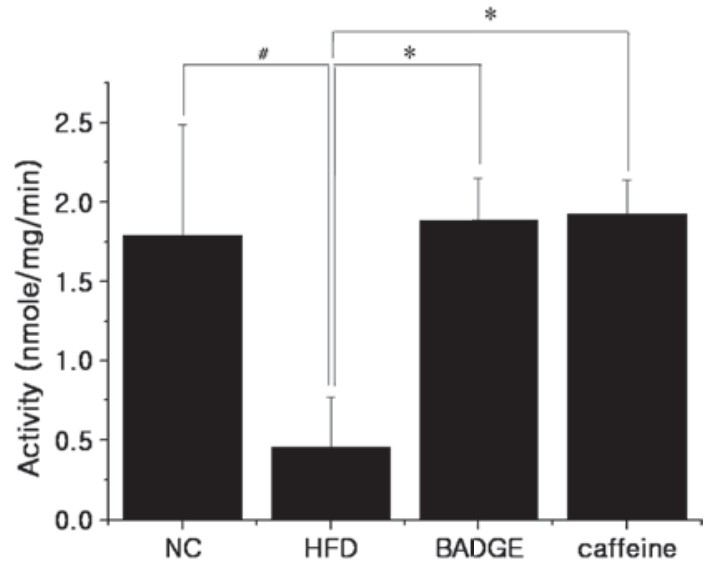

Fig. 6. Activity of CPT1 after 2 weeks of treament. NC, normal control; HFD, high-fat diet group; BADGE, HFD+BADGE group; Caffeine, HFD+caffeine group. Values represent the means $\pm \mathrm{SD}$. The means were compared by one-way ANOVA. ${ }^{\#}, P<0.05$ vs NC group. ${ }^{*}, P<0.05$ vs HFD group. BADGE, bisphenol A diglycidyl ether.

First of all, consistent with previous reports $[9,11$, 24], we found that the final body weights and the epididymal fat weights of the BADGE and the caffeine groups were significantly reduced compared to the HFD group in the C57BL/6J mice fed HFD in this study. We also demonstrated that the levels of adiponectin in epididymal white adipose tissue showed a tendency to increase in response to diets supplemented with BADGE or caffeine over the course of the experiment; especially, the increase was significant at 2 weeks of supplementation. Adiponectin, which is expressed exclusively in adipose tissue, has been shown to increase fatty acid oxidation via activation of AMPK and phosphorylation of ACC [19]. It is a secreted protein that plays a major role in the regulation of glucose, insulin and fatty acids, and functions as an anti-obesity factor [2,3,5]. Recently, it was shown that adiponectin reduces body weight in obese mice without altering food intake [10]. In HFDfed C57BL/6J mice treated with BADGE or caffeine, adiponectin expression was significantly increased after 2 weeks of supplementation, the early stage of the treatment, and it gradually decreased to the level of the HFD group. Thus, adiponectin levels may be modulated by body weight and the size of adipose tissue through a mechanism of feedback regulation. We suggest that adiponectin expression levels in adipose tissue of HFDfed C57BL/6J mice in the early stages of the BADGE or the caffeine treatment played an important role in reduced adiposity in this study. Meanwhile, the patterns of the BADGE and the caffeine response were different. The adiponection level of the caffeine group was more static between 1 and 2 weeks, compared to the BADGE group. This difference of expression patterns of adiponectin between the caffeine group and the BADGE group could be the result of different mechanisms of action of caffeine and BADGE. Hence, further studies may be necessary to elucidate the molecular mechanism of caffeine and BADGE in the regulation of adiponectin.

Fat deposits in the liver are often linked to an excess of fat mass, and in humans, generally result from increased total energy and carbohydrate intake. Evidence suggests that food restriction and lower body weight are accompanied by a reduction in liver fat in obese adults and children $[4,8,12,20]$. In the current study, histological examination of liver tissue sections revealed that HFD-fed C57BL/6J mice exhibited many abnormal appearances or histological changes including severe fatty accumulation after 8 weeks of treatment. However, we showed that in HFD-fed C57BL/6J mice, diets supplemented with BADGE or caffeine markedly reduced the number of fatty droplets in the liver.

ACC phosphorylation and CPT1 activity have been shown to be rate controlling factors in fatty acid oxidation $[14,19]$. In the current study, we also analyzed the time-course of expression levels of pACC in HFD-fed C57BL/6J mice given a diet supplemented with BADGE or caffeine. We also examined the activity of CPT1 under these same conditions. We showed that the CPT1 activity and the pACC level in liver tissues were upregulated in BADGE- and caffeine-treated mice after 2 weeks of treatment. Therefore, we suggest that BADGE and caffeine upregulate pACC and CPT1 activity in HFD-fed C57BL/6J mice, and may decrease hepatic fat accumulation via increased fatty acid oxidation. Interestingly, in our study, there were no differences in the activity of CPT1 in the BADGE and caffeine groups after 1 week of treatment. These results indicate that improvement of steatosis in BADGE- and caffeine-treated mice may correlate with increased lipid oxidation in liver tis- 
sue induced by pACC and CPT1 at an early stage of treatment, especially 2 weeks of treatment. Meanwhile, at 1 week, only the pACC level, not CPT1 activity, was increased in the BADGE group compared to the HFD group. The CPT1 activity might be indirectly regulated by ACC phosphorylation and other molecules [13]. Therefore, we showed that the CPT1 activity is not always directly proportional to the pACC level.

There are a variety of specific mechanisms by which chemicals reduce obesity. However, reductions in body weight induced by long-term treatment with anti-obesity chemicals could also be attributable to a toxic side effect that is independent of their mechanism of action. To confirm that the effects of chemicals are not due to an unexpected side-effect, and to elucidate the mechanism of action of anti-obesity chemicals, many studies have analyzed changes in the expression of adipokines or enzymes and compared these changes with body weight and adipose tissue weight changes at the endpoint of a long-term treatment regime. However, as our results show, after long-term treatment with BADGE or caffeine, body weight and adipose tissue weight in obese mice were decreased, and the expression levels of adiponectin and lipid oxidative enzymes were reduced to the levels of normal mice at the endpoint of a long-term treatment. Therefore, the analysis of the time-course response of adipokines and enzymes, including the early stages of treatment, are very important for evaluating the anti-obesity effects accurately and for elucidating the mechanism of action of anti-obesity chemicals.

In conclusion, the expression of adiponectin and lipid oxidative enzymes increased within 2 weeks of treatment with BADGE or caffeine, and the expression levels correlated well with reduced adiposity and steatosis induced by an 8 weeks of treatment in HFD-fed C57BL/6J mice.

\section{References}

1. Ahren, B. and Scheurink, A.J. 1998. Marked hyperleptinemia after high-fat diet associated with severe glucose intolerance in mice. Eur. J. Endocrinol. 139: 461-467.

2. Berg, A.H., Combs, T.P., Du, X., Brownlee, M., and Scherer, P.E. 2001. The adipocyte-secreted protein Acrp30 enhances hepatic insulin action. Nat. Med. 7: 947-953.

3. Combs, T.P., Berg, A.H., Obici, S., Scherer, P.E., and Rossetti, L. 2001. Endogenous glucose production is inhibited by the adipose-derived protein Acrp30. J. Clin. Invest. 108: 1875-1881.

4. Eriksson, S., Eriksson, K.F., and Bondesson, L. 1986. Nonalcoholic steatohepatitis in obesity: a reversible condition. Acta Med. Scand. 220: 83-88.

5. Fruebis, J., Tsao, T.S., Javorschi, S., Ebbets-Reed, D., Erickson, M.R., Yen, F.T., Bihain, B.E., and Lodish, H.F. 2001. Proteolytic cleavage product of 30-kDa adipocyte complement-related protein increases fatty acid oxidation in muscle and causes weight loss in mice. Proc. Natl. Acad. Sci. U.S.A. 98: 2005-2010.

6. Handa, T., Yamaguchi, K., Sono, Y., and Yazawa, K. 2005. Effects of fenugreek seed extract in obese mice fed a highfat diet. Biosci. Biotechnol. Biochem. 69: 1186-1188.

7. Karlic, H., Lohninger, S., Koeck, T., and Lohninger, A. 2002. Dietary $L$-carnitine stimulates carnitine acyltransferases in the liver of aged rats. J. Histochem. Cytochem. 50: 205212.

8. Keeffe, E.B., Adesman, P.W., Stenzel, P., and Palmer, R.M. 1987. Steatosis and cirrhosis in an obese diabetic. Resolution of fatty liver by fasting. Dig. Dis. Sci. 32: 441-445.

9. Kobayashi-Hattori, K., Mogi, A., Matsumoto, Y., and Takita, T. 2005. Effect of caffeine on the body fat and lipid metabolism of rats fed on a high-fat diet. Biosci. Biotechnol. Biochem. 69: 2219-2223.

10. Masaki, T., Chiba, S., Yasuda, T., Tsubone, T., Kakuma, T., Shimomura, I., Funahashi, T., Matsuzawa, Y., and Yoshimatsu, H. 2003. Peripheral, but not central, administration of adiponectin reduces visceral adiposity and upregulates the expression of uncoupling protein in agouti yellow (Ay/a) obese mice. Diabetes 52: 2266-2273.

11. Muroyama, K., Murosaki, S., Yamamoto, Y., Odaka, H., Chung, H.C., and Miyoshi, M. 2003. Anti-obesity effects of a mixture of thiamin, arginine, caffeine, and citric acid in non-insulin dependent diabetic KK mice. J. Nutr. Sci. Vitaminol. 49: 56-63.

12. Palmer, M. and Schaffner, F. 1990. Effect of weight reduction on hepatic abnormalities in overweight patients. Gastroenterology 99: 1408-1413.

13. Park, H., Kaushik, V.K., Constant, S., Prentki, M., Przybytkowski, E., Ruderman, N.B., and Saha, A.K. 2002. Coordinate regulation of malonyl-CoA decarboxylase, snglycerol-3-phosphate acyltransferase, and acetyl-CoA carboxylase by AMP-activated protein kinase in rat tissues in response to exercise. J. Biol. Chem. 277: 32571-32577.

14. Ramsay, R.R., Gandour, R.D., and van der Leij, F.R. 2001. Molecular enzymology of carnitine transfer and transport. Biochim. Biophys. Acta 1546: 21-43.

15. Reeves, P.G. 1997. Components of the AIN-93 diets as improvements in the AIN-76A diet. J. Nutr. 127: 838S-841S

16. Ruderman, N.B., Saha, A.K., Vavvas, D., and Witters, L.A. 1999. Malonyl-CoA, fuel sensing, and insulin resistance. Am. J. Physiol. 276: E1-E18.

17. Surwit, R.S., Feinglos, M.N., Rodin, J., Sutherland, A., Petro, A.E., Opara, E.C., Kuhn, C.M., and Rebuffé-Scrive, M. 1995. Differential effects of fat and sucrose on the development of obesity and diabetes in C57BL/6J and A/J 
mice. Metabolism 44: 645-651.

18. Surwit, R.S., Kuhn, C.M., Cochrane, C., McCubbin, J.A., and Feinglos, M.N. 1988. Diet-induced type II diabetes in C57BL/6J mice. Diabetes 37: 1163-1167.

19. Tomas, E., Tsao, T.S., Saha, A.K., Murrey, H.E., Zhang, Cc.C., Itani, S.I., Lodish, H.F., and Ruderman, N.B. 2002. Enhanced muscle fat oxidation and glucose transport by ACRP30 globular domain: acetyl-CoA carboxylase inhibition and AMP-activated protein kinase activation. Proc. Natl. Acad. Sci. U.S.A. 99: 16309-16313.

20. Vajro, P., Fontanella, A., Perna, C., Orso, G., Tedesco, M., and De Vincenzo,A. 1994. Persistent hyperaminotransferasemia resolving after weight reduction in obese children. J. Pediatr. 125: 239-241.

21. Vaz, F.M. and Wanders, R.J. 2002. Carnitine biosynthesis in mammals. Biochem. J. 361: 417-429.

22. West, D.B., Boozer, C.N., Moody, D.L., and Atkinson, R.L. 1992. Dietary obesity in nine inbred mouse strains. Am.J. Physiol. 262: R1025-R1032.

23. Winder, W.W. and Hardie, D.G. 1999. AMP-activated protein kinase, a metabolic master switch: possible roles in type 2 diabetes. Am. J. Physiol. 277: E1-E10.

24. Yamauchi, T., Waki, H., Kamon, J., Murakami, K., Motojima, K., Komeda, K., Miki, H., Kubota, N., Terauchi, Y., Tsuchida, A., Tsuboyama-Kasaoka, N., Yamauchi, N., Ide, T., Hori, W., Kato, S., Fukayama, M., Akanuma, Y., Ezaki, O., Itai, A., Nagai, R., Kimura, S., Tobe, K., Kagechika, H., Shudo, K., and Kadowaki, T. 2001. Inhibition of RXR and PPARgamma ameliorates diet-induced obesity and type 2 diabetes. J. Clin. Invest. 108: 1001-1013. 